\title{
Freeze-thaw risk in solid masonry: are moisture reference years able to represent real climate conditions?
}

\author{
Isabeau Vandemeulebroucke ${ }^{1,{ }^{*}}$, Steven Caluwaerts ${ }^{2}$, and Nathan Van Den Bossche ${ }^{1}$ \\ ${ }^{1}$ Department of Architecture and Urban Planning, Faculty of Engineering and Architecture, Ghent \\ University, Belgium \\ ${ }^{2}$ Department of Physics and Astronomy, Faculty of Sciences, Ghent University, Belgium
}

\begin{abstract}
Today, there is no consensus on the selection method of representative exterior boundary conditions when performing HAM (Heat Air Moisture) simulations on building envelopes. Many existing methods to select moisture reference years (MRY) fail to provide an acceptable validation in terms of quantified risk assessment. Although new methods have been suggested during the past few years, the influence of several parameters on the selection of "critical years" in long-term datasets still needs to be assessed. The objective of this paper is to validate the application of MRY's to evaluate freeze-thaw risk in retrofitted solid masonry. Furthermore, the influence of the chosen wall assembly, damage criterion, preconditioning and start date of the evaluation period on the ranking of critical years is assessed, using a 31-year meteorological dataset of Brussels. Results indicate that for a given wall assembly and freezethaw criterion, as well as a smart start date of the evaluated period, single year simulations entail a similar ranking of critical years as the corresponding year in the 31-year simulation. The number of critical freeze-thaw cycles only varies between 0 - 2 cycles $(0-2.9 \%)$. However, changing the wall assembly and damage criterion, alters the top 5 ranking of critical years substantially.
\end{abstract}

\section{Introduction}

Moisture is a predominant agent of building envelope deterioration [1]-[3]. Common damage mechanisms in building facades are mould growth, wood decay, freeze-thaw damage, erosion, corrosion etc. However, moisture related damage often only arises when the moisture balance is disturbed, leading to the accumulation of moisture in certain building components. To evaluate the moisture behaviour of the building envelope, HAM simulations are performed. Along with the definition of the wall configuration, material properties, boundary conditions and wall orientation, a representative outdoor climate needs to be defined to evaluate the long-term durability of the wall assembly.

\footnotetext{
* Corresponding author: isabeau.vandemeulebroucke@ugent.be
} 
HAM simulations are generally conducted for a series of one or more moisture reference years (MRY) to limit simulation time and memory. Today there is no consensus on the selection or synthetization method for MRY's, referred to as MRY-method. Since the end of the $20^{\text {th }}$ century, MRY-methods have been proposed, however none of them yet succeeded in providing one MRY to answer all moisture and heat related questions. When analysing literature, some general agreements concerning the MRY-method can be distinguished. Firstly, the originating dataset for MRY's should be of sufficient length, generally 30 years, to take into account climate variability and return periods of climate conditions. Secondly, the MRY should be location-specific and provide a good summary of the outdoor climate. Additionally, the MRY should allow for the evaluation of the hygrothermal response under critical moisture stress. Generally, the MRY is a 1/10 level year, i.e. having a return period of 10 years.

On the other hand, many criteria in the MRY-method remain inconclusive: it is unclear whether the method should be strictly construction-dependent, or whether a constructionindependent method might provide representative MRY's. Furthermore, a variety of key indicators to select MRY's have been proposed: mean air temperature, relative humidity, precipitation, mould index, drying and/or wetting potential, RHT index etc. [3]-[9]. Besides, existing methodologies often only relate to one specific damage mechanism (e.g. mould growth or condensation risk), which might render the selected critical year inapt for other degradation processes.

Although freeze-thaw damage is an important deteriorating agent in many (retrofitted) heritage buildings, to the authors' knowledge no MRY-method focusses specifically on freeze-thaw risk in porous media. Besides, freeze-thaw damage is no robust damage mechanism. A high wind-driven rain (WDR) load during winter or cold temperatures do not necessarily induce more freeze-thaw damage. The occurrence of critical freeze-thaw cycles $\left(\mathrm{FTC}_{\text {crit }}\right)$, however, strongly depends on the sequence of weather events, such as the freezing rate following WDR events, WDR intensity and duration, drying rate etc. [10]. The wrong MRY could entail misleading conclusions on freeze-thaw risk, and therefore it is important to properly select reference years.

The objective of this paper is to validate the application of MRY's to evaluate freezethaw risk in retrofitted solid masonry. This is achieved by comparing single-year simulation results to the corresponding year in a long-term simulation, based on meteorological measurements in Brussels (BE). Furthermore, the study aspires to highlight and analyse the influence of different parameters on the selection of MRY's, such as wall assembly, damage criterion, preconditioning and start date of the evaluation period. The paper is organized as follows: in section two the methodology is discussed in terms of the meteorological conditions, wall assemblies and reference case. Section three focusses on the results and discussion: the influence of the chosen wall assembly and damage criterion on the top 5 ranking of critical years is evaluated, the usage of single-year simulations to represent long-term simulation results is validated, and the importance of preconditioning and start date of the evaluation period is assessed. The conclusions are formulated in section four.

\section{Methodology}

The hygrothermal simulations are performed in Delphin 5.9.5, successfully validated in the past through HAMSTAD Benchmark exercises 1-5 e.g. [11]. 


\subsection{Meteorological conditions}

Measured meteorological data of a 31-year period (1987-2017) in Brussels (BE) are used as exterior climate conditions in HAM simulations. The meteorological dataset contains hourly data for air temperature, relative humidity, precipitation, wind velocity and direction at $10 \mathrm{~m}$ height, cloud cover, and direct and diffuse solar radiation. Downward longwave radiation is taken into account using the calculated sky temperature, whereas the ground temperature, considered equal to the air temperature, is used in the equation for upward longwave radiation.

\subsection{Wall assembly}

Two wall assemblies are evaluated in this study, i.e. a solid masonry wall assembly and an internally retrofitted solid masonry wall assembly. The first configuration consists of 300 $\mathrm{mm}$ of historical brick (cluster 4 in Delphin) and $12 \mathrm{~mm}$ of gypsum plaster, whereas the second wall assembly is composed of $300 \mathrm{~mm}$ masonry of the same kind, $150 \mathrm{~mm}$ mineral wool, a vapour barrier and $12 \mathrm{~mm}$ gypsum board. Material properties are originating from the Delphin Material Library. The masonry is considered homogenous, a simplification found valid by Vereecken and Roels [12].

\subsection{Reference case}

The reference case in this paper is the retrofitted solid masonry wall, exposed to measured exterior conditions in Brussels (1987-2017), and preceded by four conditioning years. The conditioning years are equal to four times the year 1987, i.e. the first year of the dataset, and are not considered during evaluation. The criterion to count the number of $\mathrm{FTC}_{\text {crit }}$ is a critical degree of moisture saturation $\left(\mathrm{S}_{\text {crit }}\right)$ of 0.25 . Meaning that for the considered type of brick, the critical ice mass density is $82.5 \mathrm{~kg} / \mathrm{m}^{3}$.

\section{Results and discussion}

\subsection{Wall assembly}

Although some researchers define MRY's only based on meteorological conditions, others believe that reference years cannot be determined independently from the wall assembly [3]-[9]. To study the influence of the wall assembly on the number of $\mathrm{FTC}_{\text {crit }}$, two wall assemblies are considered. An overview of the top 5 ranking of critical years for different variations of the reference case is reported in table 1 . The ranking of critical years is important, given that MRY's commonly have a 10-year return period. Based on a 31-year dataset, the MRY should be the third ranked year. Table 1 illustrates that the number of $\mathrm{FTC}_{\text {crit }}$ is generally higher after retrofitting the solid masonry than in the original wall. The maximum number of $\mathrm{FTC}_{\text {crit }}$ increases from 34 to 62 . Besides, $\mathrm{FTC}_{\text {crit }}$ reach twice as deep in the masonry, from the middle of the masonry before retrofitting to the interior masonry surface after retrofitting. When comparing the ranks of descending number of $\mathrm{FTC}_{\text {crit, }}$, all different years are listed in the top 5 (table 1). It can be concluded that the wall assembly has a major influence on the choice of critical years based on number of FTC $_{\text {crit }}$. 


\subsection{Damage criterion}

Apart from the choice of damage mechanism to evaluate the durability of building materials, also the preselected damage criterion influences the assessed risk on deterioration. In the case of freeze-thaw related damage, the critical degree of moisture saturation $\left(\mathrm{S}_{\text {crit }}\right)$ is known to vary between 0.25 and 0.90 for different types of brick masonry [13], [14]. Especially when the $S_{\text {crit }}$ of the particular masonry is not known, the critical value is typically set conservatively. When assessing the critical years from longterm meteorological data based on the number of $\mathrm{FTC}_{\text {crit }}$, simulation results illustrate that different criteria entail different top 5 rankings (table 1). Between the results having a freeze-thaw criterion 0.25 and 0.9 there is only one mutual year in the ranking, i.e. 2004. The freeze-thaw criterion 0.5 shares one or two years with both of the former criteria. In the case of the uninsulated solid masonry wall assembly having a $S_{\text {crit }}$ of 0.9 (not illustrated in this paper), no $\mathrm{FTC}_{\text {crit }}$ occur across the entire long-term dataset. It can be concluded that the freeze-thaw criterion has a major influence on the selection of critical years in a 31-year dataset. Whereas a low freeze-thaw criterion, rather on the safe side, might bias the selection of critical years, a high freeze-thaw criterion may not lead to any critical year when the criterion is never reached.

Table 1. Top 5 ranking based on descending number of critical freeze-thaw cycles (the number of $\mathrm{FTC}_{\text {crit }}$ is indicated between brackets)

\begin{tabular}{|c|c|c|c|c|c|c|}
\hline & Variation & Rank 1 & Rank 2 & Rank 3 & Rank 4 & Rank 5 \\
\hline $\mathrm{L}$ & Reference & $2006(62)$ & $1987(58)$ & $2008(50)$ & 2009 (48) & $2004(45)$ \\
\hline $\mathrm{L}$ & $\begin{array}{l}\text { Uninsulated } \\
\text { masonry }\end{array}$ & $1991(34)$ & $1992(16)$ & 2007 (14) & $\begin{array}{l}1999(13) \\
2012 \\
2015\end{array}$ & / \\
\hline $\mathrm{L}$ & $\mathrm{S}_{\text {crit }}=0.50$ & $\begin{array}{l}1991(41) \\
2013\end{array}$ & 1 & $\begin{array}{l}1987 \text { (39) } \\
2006\end{array}$ & / & $2001(36)$ \\
\hline $\mathrm{L}$ & $\mathrm{S}_{\text {crit }}=0.90$ & $1991(31)$ & $1992(28)$ & $1993(23)$ & $\begin{array}{l}1999 \text { (17) } \\
\mathbf{2 0 0 4}\end{array}$ & / \\
\hline $\mathrm{S}$ & Single years & $2006(63)$ & $1996(62)$ & $1987(58)$ & $2008(49)$ & 2009 (48) \\
\hline $\mathrm{L}$ & $01 / 09-31 / 08$ & $2005-06(69)$ & $2008-09(61)$ & $2012-13(50)$ & 1990-91 (44) & $\begin{array}{l}2002-03 \\
(43)\end{array}$ \\
\hline $\mathrm{S}$ & $01 / 09-31 / 08$ & $2005-06(70)$ & $2008-09(59)$ & $2013-14(52)$ & $\begin{array}{l}1990-91(43) \\
2009-10 \\
2014-15\end{array}$ & 1 \\
\hline
\end{tabular}

L: long-run (1987-2017); S: single-year run; $\mathrm{S}_{\text {crit }}=$ critical degree of moisture saturation

\subsection{Single-year simulation}

In HAM simulation, it is common practice to use one reference year both as the conditioning and evaluation year. However, this may lead to an unrealistic sequence of meteorological years. In this section, single-year simulations are performed for each of the 31 individual years. The year is repeated 5 times, e.g. 5 times 1987 , and the $5^{\text {th }}$ year is compared to the corresponding year in the long-term simulation (1987-2017). The difference in number of $\mathrm{FTC}_{\text {crit }}$ ranges between 0 and 5 in most cases. During the year 1996, however, the difference is $36 \mathrm{FTC}_{\text {crit. }}$ When repeating the year 1996 in the single-year simulation, the moisture content between January and May is significantly higher than under real, historic weather conditions, and the $\mathrm{S}_{\text {crit }}$ concurrently with freezing temperatures is reached more often leading to a higher occurrence of $\mathrm{FTC}_{\text {crit }}$ (figure 1). From June onwards, the difference in moisture content and number of $\mathrm{FTC}_{\text {crit }}$ disappears. Hereby, the influence of the conditioning year and start date of the evaluation period is illustrated, discussed further in section 3.4 and 3.5. Although, for many years a good resemblance 
between the number of $\mathrm{FTC}_{\text {crit }}$ is achieved from single-year simulations, they are not always appropriate to represent real moisture conditions (when the evaluation period coincide with a calendar year).
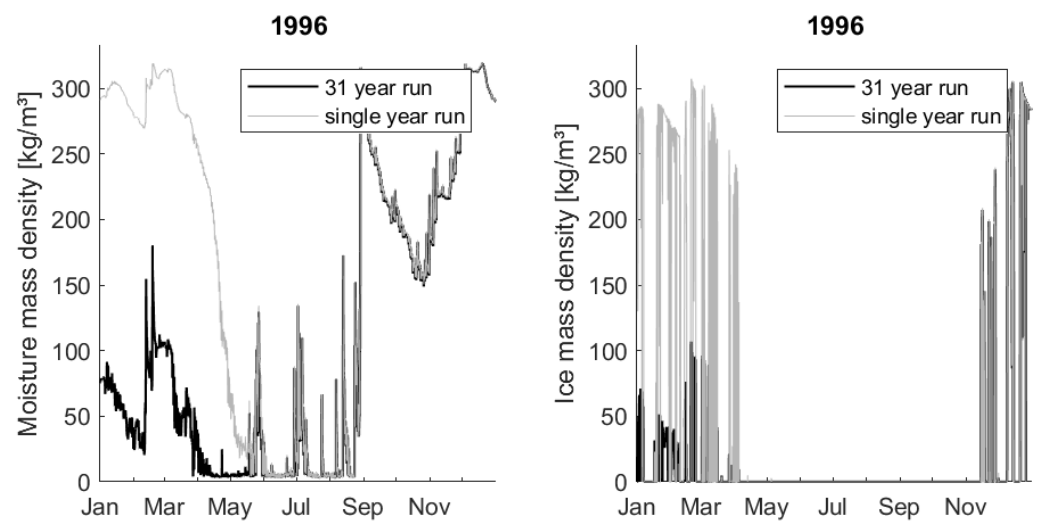

Fig. 1. The moisture content (left) and ice mass density (right) at $5 \mathrm{~mm}$ in the masonry (exterior).

\subsection{Conditioning}

Conditioning the wall assembly is an important aspect in HAM simulations as it allows to achieve an equilibrium condition for moisture content in the construction prior to simulating the actual period of interest, referred to as the evaluation year(s). The duration of conditioning depends on the wall assembly and material properties, as well as the exterior boundary conditions. In this study, four years of preconditioning is found to be a sufficient period.

Figure 1 illustrates that the influence of the conditioning year is limited to the first months of the evaluation year. For the long-term simulation, it suffices to check whether the first year of the dataset can be used to condition the wall assembly. Based on the results of the reference case, a 'dry', 'average' and 'wet' year are selected in terms of annual average moisture content, respectively 1995, 2015 and 1991. Three simulations are performed using each of these years for preconditioning, followed by the evaluation year 1987. The results indicate that the number of $\mathrm{FTC}_{\text {crit }}$ in 1987 ranges between 61-64, but the position of the year in the top 5 ranking remains identical. Please note that it is dependent on the specific dataset whether the first year can be used for preconditioning.

\subsection{Start date of the evaluation period}

Hygrothermal simulations commonly start at January 1 th, however there are studies that indicate that a MRY should not coincide with a calendar year [3], [7]. Because autumn, winter and spring are typically the critical seasons in terms of moisture related damage, the sequence of these seasons probably should be considered in its integrity to avoid discontinuities in the period of interest. Because the risk on internal condensation and mould growth is usually the lowest during summer (e.g. in Estonia), Kalamees and Vinha [7] suggest that a MRY starts at July 1th. Zhou et al. [3] propose October 1th, in accordance with the start of the 'wet' period of the evaluation year (e.g. in Switzerland), also referred to as a water year [15].

In this section the number of $\mathrm{FTC}_{\text {crit }}$ during a calendar year and a year starting at September 1th are compared. Table 1 indicates that for the reference case (calendar year) 
the years 2006, 1987 and 2008 are ranked highest, whereas in the other case (years starting at September 1th) the periods 2005-06 (01/09/2005 - 31/08/2006), 2008-09 and 2012-13 are on top of the list of number of $\mathrm{FTC}_{\text {crit }}$. This illustrates the overlap between some critical years, but not all of them. When comparing the number of $\mathrm{FTC}_{\text {crit }}$ in the top 5 ranking, the difference ranges between $0 \%$ and $11.3 \%$. In the uninsulated case (not illustrated in this paper), the difference ranges between $7.7 \%$ and $41.7 \%$.

When comparing the single-year simulations to the long-term simulation with the evaluation years starting at September 1th, the difference in number of $\mathrm{FTC}_{\text {crit }}$ ranges between 0 and 2 cycles $(2.9 \%)$. The results of these single-year simulations are closer to the real situation than when calendar years are considered. Note that the large difference in the number of $\mathrm{FTC}_{\text {crit }}$ during the year 1996 entirely dissolves (please refer to section 3.3). There is no improvement for an evaluation year starting at June 1th. Please note that the start date of the evaluation years coincides with the meteorological seasons to maintain continuity within each season.

\section{Conclusion}

It can be concluded that the application of single-year simulations are appropriate to represent the freeze-thaw risk of the corresponding year in long-term simulations. However, this is only valid for a given wall assembly and freeze-thaw criterion, as these aspects have a major impact on the ranking of critical years. Furthermore, the evaluation period should not coincide with a calendar year, but start outside the potential freezing period (e.g. September 1th in the case of Brussels).

The authors would like to acknowledge the Royal Meteorological Institute of Belgium for providing the long-term measurements of meteorological data.

\section{References}

1. J. Lstiburek, ASHRAE J. 2, pp. 36 (2002)

2. J. Straube, C. Schumacher, J. Green Build. 2, 2 (2007)

3. X. Zhou, D. Derome, J. Carmeliet, Build. Environ. 110, pp. 23-35 (2016)

4. F. Ali Mohamed, H. Hens, Annex 24 HAMTIE (1992)

5. C.-E. Hagentoft, E. Harderup, Annex 24 HAMTIE (1996)

6. S. Geving, Moisture Design of Building Constructions: Hygrothermal analysis using simulation models - Part I and II (NTNU, 1997)

7. T. Kalamees, J. Vinha, J. Build. Phys. 27, 3 (2004)

8. M. Salonvaara, K. Sedlbauer, A. Holm, M. Pazera, Proc. Therm. Perform. Exter. Envel. Whole Build. XI Int. Conf. (2010)

9. V. M. Nik, Energy Build. 154, pp. 30-45 (2017)

10. I. Vandemeulebroucke, K. Calle, S. Caluwaerts, T. De Kock, N. Van Den Bossche, Can. J. Civ. Eng. (published online 2019)

11. A. Nicolai, J. Grunewald, J. J. Zhang, Conf. Proc. 12th Symp. Build. Phys. (2007)

12. E. Vereecken and S. Roels, Constr. Build. Mater. 41, pp. 697-707 (2013)

13. G. Fagerlund, Conf. Proc. Durab. Concr. ACI STP, Atl. 17, pp. 13-65 (1975)

14. P. Mensinga, J. Straube, C. Schumacher, Proc. Performances Envel. Whole Build. XI (2010) 
15. R. S. V. Teegavarapu, A. Nayak, J. Hydrol. 550, pp. 616-634 (2017) 